\title{
SINGULAR PERTURBATION SOLUTION OF A CLASS OF SINGULAR INTEGRAL EQUATIONS
}

\author{
BY \\ J. R. WILLIS (University of Bath, Bath, U.K.) \\ AND \\ S. NEMAT-NASSER (University of California, San Diego, La Jolla, California)
}

\begin{abstract}
A formal method is developed for finding asymptotic solutions to a class of strongly singular integral equations containing a small parameter, $\varepsilon$. The class has relevance to the analysis of microcrack growth in reinforced ceramics. The method makes use of the asymptotic matching principle of Van Dyke. Its application is mechanical and it appears to allow, in principle, the construction of asymptotic solutions to any order. Consistency to order $\varepsilon$ is demonstrated for the general case and a solution correct to order $\varepsilon^{2}$ is constructed for a particular example, previously studied only to leading order.
\end{abstract}

1. Introduction. There has recently been substantial interest in the asymptotic solution of integral equations containing a small parameter, in cases where the parameter gives rise to a singular perturbation. Systematic studies of Volterra equations have been made by Angell and Olmstead [1, 2] and Fredholm equations have been discussed by Lange and Smith [3]. For these examples, solutions could be generated, in principle to any order, starting from a simple "additive" form of asymptotic approximation. For other types of equations, "leading order" asymptotic solutions can often be found. Atkinson and Leppington [4], for example, studied two equations (one with a Cauchy kernel) and extracted solutions by direct reasoning. Hori and Nemat-Nasser [5] studied an equation with a strongly-singular kernel and found a leading-order solution, both by starting from an "additive" form and a "multiplicative" form for the solution. Neither starting-point appeared capable, however, of yielding a solution to higher order — or at least some further injection of ingenuity appeared to be required. Olmstead and Gautesen [6] have likewise obtained leadingorder solutions to a variety of equations, starting from a "multiplicative" form for the solution.

The purpose of this work is to develop a simple prescription, which can be applied directly to obtain the asymptotic solution, in principle to any order, of equations of the type considered by Hori and Nemat-Nasser [5]. The approach has much in

Received October 25, 1989.

(C)1990 Brown University 
common with that developed in [1,2,3] but the question of matching expansions is addressed in much the way employed for differential equations by Van Dyke [7].

In the singular perturbation of differential equations, formal "inner" and "outer" asymptotic expansions may be generated. Loss of boundary conditions gives rise to undetermined parameters in the expansions, which may then be fixed by "matching." One particular technique for matching (which is invoked in the present work) is embodied in the asymptotic matching principle of Van Dyke [7]. As noted in [1, 2, 3], integral equations are different in that a uniformly valid asymptotic approximation has to be substituted directly into the integral. The "additive," or "multiplicative," forms are intended to serve that purpose. In the present work, this idea is extended - or perhaps expressed in a way that makes its application routine - by introducing explicitly a composite expansion, comprising the sum of inner and outer expansions, less their common part. The matching principle is thus invoked at an earlier stage than in the case of differential equations.

Inevitably, the application of the method becomes increasingly laborious, the more terms are required, but, for illustration, the procedure is carried through to three terms for a particular linear equation, studied to leading order in [5] and [6].

2. The integral equation. The integral equation

$$
-\varepsilon \int_{-1}^{1} \frac{d y}{(y-x)^{2}} \varphi(y)+\gamma(x, \varphi(x))=\psi(x), \quad-1<x<1,
$$

together with the subsidiary conditions

$$
\varphi(-1)=\varphi(1)=0,
$$

is considered, in the asymptotic limit $\varepsilon \ll 1$. It was studied previously by Hori and Nemat-Nasser [5], who demonstrated uniqueness of its solution (assuming existence), under the assumption that $\gamma$ is a monotone increasing function of its second argument, $\varphi$. The parameter $\varepsilon$ is positive. The integral in $(2.1)$ is strongly singular and is interpreted as a finite part in the sense of Hadamard [8] or, equivalently, in the sense of distributions [9]. Thus, it can be viewed as the convolution of $\varphi(x)$ (taken equal to zero outside $[-1,1]$ ) with $x^{-2}$. A simple way to evaluate the convolution is to regard $x^{-2}$ as the real part of $(x-0 i)^{-2}$, since the integral can be evaluated by elementary means for the latter. The result is that

$$
\begin{aligned}
\int_{-1}^{1} \frac{d y}{(y-x)^{2}} \varphi(y)= & \int_{-1}^{1} \frac{d y}{(y-x)^{2}}\left[\varphi(y)-\varphi(x)-(y-x) \varphi^{\prime}(x)\right] \\
& -\frac{2 \varphi(x)}{1-x^{2}}+\varphi^{\prime}(x) \ln \left(\frac{1-x}{1+x}\right) .
\end{aligned}
$$

Equation (2.3) makes sense so long as $\varphi^{\prime}(x)$ is defined; it is useful when, in addition, $\varphi(x)$ is smooth, so that the remaining integrand has only a removable singularity at $y=x$.

One physical context in which the integral equation (2.1), with (2.2), appears, is in the analysis of a crack in a brittle matrix reinforced by fibres [10]. The crack occupies (in dimensionless variables) the segment $-1<x<1$ of the $x$-axis and the external 
loading, defined by $\psi(x)$, induces a separation $\varphi(x)$ of the crack faces. The crack is bridged by fibres, which exert a restraining stress at $x$ equal to $\gamma(x, \varphi(x))$, which thus depends on the local separation. If all fibres are broken at some particular $x$, then $\gamma(x, \varphi(x))=0$. The integral corresponds to the resistance to crack opening provided by the elasticity of the matrix. Thus, the limit $\varepsilon \ll 1$ is associated with the dominance of crack bridging over the elasticity of the matrix. The value of $\varepsilon$ decreases as the physical length of the crack increases.

The limit $\varepsilon \ll 1$ is singular because, if $\varepsilon$ were set equal to zero in (2.1), the predicted $\varphi(x)$ would violate the conditions (2.2); thus, the integral has to be important, at least near $x= \pm 1$. For future use, the asymptotic form of (2.3), when $x=-1+\varepsilon x^{\prime}$ and $\varepsilon \rightarrow 0$ with $x^{\prime}$ fixed, is recorded:

$$
\begin{aligned}
\int_{-1}^{1} \frac{d y}{\left(y+1-\varepsilon x^{\prime}\right)^{2}} \varphi(y) \sim & \int_{-1}^{1} \frac{d y}{(y+1)^{2}}\left[\varphi(y)-\varphi(-1)-(y+1) \varphi^{\prime}(-1)\right] \\
& -\frac{\varphi(-1)}{\varepsilon x^{\prime}}-\frac{1}{2} \varphi(-1)-\varphi^{\prime}(-1) \\
& +\varphi^{\prime}(-1) \ln \left(\frac{2}{\varepsilon x^{\prime}}\right)+o(1) .
\end{aligned}
$$

3. The matching procedure. Suppose that

$$
\varphi(x) \sim \varphi_{0}(x)+\varphi_{1}(x)+\cdots+\varphi_{n}(x)+o\left(\varepsilon^{n}\right) \quad \text { as } \varepsilon \rightarrow 0,
$$

with $x$ fixed and different from -1 or 1 , and that

$$
\varphi\left(-1+\varepsilon x^{\prime}\right) \sim \tilde{\varphi}_{0}\left(x^{\prime}\right)+\tilde{\varphi}_{1}\left(x^{\prime}\right)+\cdots+\tilde{\varphi}_{m}\left(x^{\prime}\right)+o\left(\varepsilon^{m}\right) \quad \text { as } \varepsilon \rightarrow 0,
$$

with $x^{\prime}$ fixed. For this purpose, $\ln \varepsilon$ is regarded as $O(1)$. The function $\varphi(x)$ is assumed to satisfy the asymptotic matching principle of Van Dyke [7]. That is, if

$$
\varphi_{k}\left(-1+\varepsilon x^{\prime}\right) \sim S_{k}^{m}\left(x^{\prime}\right)+o\left(\varepsilon^{m}\right) \quad \text { as } \varepsilon \rightarrow 0
$$

and

$$
\tilde{\varphi}_{k}\left(\frac{1+x}{\varepsilon}\right) \sim \tilde{S}_{k}^{n}(x)+o\left(\varepsilon^{n}\right) \quad \text { as } \varepsilon \rightarrow 0,
$$

then

$$
\sum_{k=0}^{n} S_{k}^{m}\left(\frac{1+x}{\varepsilon}\right)=\sum_{k=0}^{m} \widetilde{S}_{k}^{n}(x),
$$

exactly. In this case, it is assumed that

$$
\varphi(x) \sim \sum_{k=0}^{n}\left(\varphi_{k}(x)+\tilde{\varphi}_{k}\left(x^{\prime}\right)-S_{k}^{n}\left(x^{\prime}\right)\right)+o\left(\varepsilon^{n}\right)
$$

uniformly for $-1 \leq x<1-\delta$ for some $\delta>0$. Here,

$$
x^{\prime}=\frac{1+x}{\varepsilon} .
$$

A similar assumption will be made for the other end-point, $x=1$. The decomposition (3.6) is additive, like that used in [1,2,3], but the explicit inclusion of the terms 
$S_{k}^{n}\left(x^{\prime}\right)$, which can equally well be written in terms of $x$, using (3.5), is helpful for the calculations to follow.

The notation introduced above is such that $\varphi_{k}$ and $\tilde{\varphi}_{k}$ contain only terms of order $\varepsilon^{k}$ (including $\varepsilon^{k} \ln \varepsilon$, etc.) but $S_{k}^{n}, \widetilde{S}_{k}^{n}$ denote series containing terms from $O($ l $)$ up to $O\left(\varepsilon^{n}\right)$.

The procedure is now to attempt to find $\varphi_{k}, \tilde{\varphi}_{k}$ by substituting the uniform expansion (3.6) into the integral in (2.1) and then simplifying the resulting expressions, either as $\varepsilon \rightarrow 0$ with $x$ fixed or as $\varepsilon \rightarrow 0$ with $x^{\prime}$ fixed, $x$ and $x^{\prime}$ being related by (3.7).

4. Solution to lowest order. First, let $\varepsilon \rightarrow 0$ in (2.1), with $x$ fixed. Eq. (2.3) shows that the integral is bounded, so $(2.1)$ reduces to

$$
\gamma\left(x, \varphi_{0}(x)\right)=\psi(x)
$$

which is an algebraic equation for $\varphi_{0}(x)$. Close to $x=-1$, however, the integral becomes important. It will be approximated by using the expression (3.6), with $n=0$. This requires knowledge of $S_{0}^{0}\left(x^{\prime}\right)$, which follows immediately as

$$
S_{0}^{0}\left(x^{\prime}\right)=\varphi_{0}(-1) \text {. }
$$

Now $x$ is identified with $-1+\varepsilon x^{\prime}$, as in (3.7), and (2.1) is expanded to lowest order as $\varepsilon \rightarrow 0$. The equation to be analyzed is

$$
\begin{aligned}
& -\varepsilon \int_{-1}^{1} \frac{d y}{(y-x)^{2}}\left[\varphi_{0}(y)+\tilde{\varphi}_{0}\left(y^{\prime}\right)-\varphi_{0}(-1)\right]+\gamma\left(-1+\varepsilon x^{\prime}, \tilde{\varphi}_{0}\left(x^{\prime}\right)\right) \\
& \quad \sim \psi\left(-1+\varepsilon x^{\prime}\right),
\end{aligned}
$$

in which $y^{\prime}=\frac{(1+y)}{\varepsilon}$. The result (2.3) shows that

$$
\varepsilon \int_{-1}^{1} \frac{d y}{\left(y+1-\varepsilon x^{\prime}\right)^{2}}\left[\varphi_{0}(y)-\varphi_{0}(-1)\right]=O(\varepsilon)
$$

and so can be ignored. The integral involving $\tilde{\varphi}_{0}\left(y^{\prime}\right)$ is expanded as follows:

$$
\varepsilon \int_{-1}^{1} \frac{d y}{(y-x)^{2}} \tilde{\varphi}_{0}\left(y^{\prime}\right)=\int_{0}^{\infty} \frac{d y^{\prime}}{\left(y^{\prime}-x^{\prime}\right)^{2}} \tilde{\varphi}_{0}\left(y^{\prime}\right)-\int_{2 / \varepsilon}^{\infty} \frac{d y^{\prime}}{\left(y^{\prime}-x^{\prime}\right)^{2}} \tilde{\varphi}_{0}\left(y^{\prime}\right) .
$$

When $x^{\prime}>\frac{2}{\varepsilon}, \tilde{\varphi}_{0}\left(x^{\prime}\right)$ can be replaced by $\tilde{S}_{0}^{0}\left(-1+\varepsilon x^{\prime}\right)$, which equals $\varphi_{0}(-1)$, from the matching principle (3.5). Evaluating the last integral in (4.5) then gives

$$
\varepsilon \int_{-1}^{1} \frac{d y}{(y-x)^{2}} \tilde{\varphi}_{0}\left(y^{\prime}\right) \sim \int_{0}^{\infty} \frac{d y^{\prime}}{\left(y^{\prime}-x^{\prime}\right)^{2}} \tilde{\varphi}_{0}\left(y^{\prime}\right)+O(\varepsilon) .
$$

Hence, to lowest order, (4.3) gives

$$
-\int_{0}^{\infty} \frac{d y^{\prime}}{\left(y^{\prime}-x^{\prime}\right)^{2}} \tilde{\varphi}_{0}\left(y^{\prime}\right)+\gamma\left(-1, \tilde{\varphi}_{0}\left(x^{\prime}\right)\right)=\psi(-1) .
$$

This equation was also derived by Hori and Nemat-Nasser [5]. Its solution is nontrivial and can be addressed only through particular examples. It can be deduced 
directly from (4.7), however, that, as $x^{\prime} \rightarrow \infty$, the integral approaches zero, while $\tilde{\varphi}_{0}\left(x^{\prime}\right)$ can be replaced by $\widetilde{S}_{0}^{0}(x)$. Thus,

$$
\gamma\left(-1, \widetilde{S}_{0}^{0}(x)\right)=\psi(-1)
$$

confirming that $\tilde{S}_{0}^{0}=S_{0}^{0}=\varphi_{0}(-1)$, as required for consistency with the matching principle (3.5).

5. Solution to first order. Proceeding in a similar way, terms of order $\varepsilon$ are now retained in (2.1), with $x$ held fixed. This time, a uniform zeroth-order expression for the integrand will make a contribution. Thus,

$$
\begin{aligned}
& -\varepsilon \int_{-1}^{1} \frac{d y}{(y-x)^{2}}\left\{\varphi_{0}(y)+\left[\tilde{\varphi}_{0}\left(\frac{1+y}{\varepsilon}\right)-\varphi_{0}(-1)\right]+\cdots\right\} \\
& \quad+\gamma\left(x, \varphi_{0}(x)+\varphi_{1}(x)\right) \sim \psi(x) .
\end{aligned}
$$

The terms not given explicitly come from the solution near $y=1$. To order $\varepsilon$, the contributions from near $y=-1$ and $y=1$ are in any case zero. Considering $y$ close to -1 , the term in square brackets in the integrand is $O(\varepsilon)$, except for a region within a distance of $O(\varepsilon)$ of $y=-1$. Its integral is therefore $O(\varepsilon)$ and this is multiplied by $\varepsilon$. Thus, (5.1) gives

$$
\varphi_{1}(x) \gamma_{, \phi}\left(x, \varphi_{0}(x)\right)=\varepsilon \int_{-1}^{1} \frac{d y}{(y-x)^{2}} \varphi_{0}(y)
$$

having taken into account (4.1).

To progress further, an expression correct to $O(\varepsilon)$ for the integrand is required, and this involves $S_{0}^{1}\left(x^{\prime}\right), S_{1}^{1}\left(x^{\prime}\right)$. First,

$$
S_{0}^{1}\left(x^{\prime}\right)=\varphi_{0}(-1)+\varepsilon x^{\prime} \varphi_{0}^{\prime}(-1) \text {. }
$$

It may be noted also that

$$
\varphi_{0}^{\prime}(-1)=\left[\psi(-1)-\gamma_{, x}\left(-1, \varphi_{0}(-1)\right] / \gamma_{, \varphi}\left(-1, \varphi_{0}(-1)\right) .\right.
$$

The other function, $S_{1}^{1}\left(x^{\prime}\right)$, is obtained by setting $x=-1+\varepsilon x^{\prime}$ in (5.2) and expanding. This gives

$$
S_{1}^{1}\left(x^{\prime}\right) \gamma_{, \varphi}\left(-1+\varepsilon x^{\prime}, S_{0}^{1}\left(x^{\prime}\right)\right) \sim-\frac{\varphi_{0}(-1)}{x^{\prime}}+\varepsilon \varphi_{0}^{\prime}(-1) \ln \left(\frac{2}{\varepsilon x^{\prime}}\right)+\varepsilon A,
$$

where

$$
A=\int_{-1}^{1} \frac{d y}{(y-1)^{2}}\left[\varphi_{0}(y)-\varphi_{0}(-1)-(y+1) \varphi_{0}^{\prime}(-1)\right]-\frac{1}{2} \varphi_{0}(-1)-\varphi_{0}^{\prime}(-1),
$$

having used (2.4). Hence, expanding also the term $\gamma_{, \varphi}$,

$$
\begin{aligned}
S_{1}^{1}\left(x^{\prime}\right) \gamma_{, \varphi}\left(-1, \varphi_{0}(-1)\right)= & -\frac{\varphi_{0}(-1)}{x^{\prime}}+\varepsilon \varphi_{0}^{\prime}(-1) \ln \left(\frac{2}{\varepsilon x^{\prime}}\right)+\varepsilon A \\
& +\varepsilon \varphi_{0}(-1)\left[\gamma_{, x \varphi}+\varphi_{0}^{\prime}(-1) \gamma_{, \varphi \varphi}\right] / \gamma_{, \varphi},
\end{aligned}
$$

where $\gamma_{, x \varphi}$ etc. are evaluated at $\left(-1, \varphi_{0}(-1)\right)$. 
The forms $\tilde{S}_{0}^{1}(x), \tilde{S}_{1}^{1}(x)$ are also of interest. To find $\tilde{S}_{0}^{1}(x), x^{\prime}=(1+x) / \varepsilon$ is substituted into (4.7), to yield

$$
-\varepsilon \int_{-1}^{\infty} \frac{d y}{(y-x)^{2}} \tilde{\varphi}_{0}\left(\frac{1+y}{\varepsilon}\right)+\gamma\left(-1, \widetilde{S}_{0}^{1}(x)\right) \sim \psi(-1) .
$$

In the integral, $\tilde{\varphi}_{0}$ differs from $\varphi_{0}(-1)$ by a term of order $\varepsilon$, except within a distance of order $\varepsilon$ of $y=-1$. It can be approximated, therefore, by replacing $\tilde{\varphi}_{0}$ by $\varphi_{0}(-1)$. Evaluating the integral and expanding $\gamma$ then gives

$$
\left[\widetilde{S}_{0}^{1}(x)-\varphi_{0}(-1)\right] \gamma_{, \varphi}\left(-1, \varphi_{0}(-1)\right)=-\frac{\varepsilon \varphi_{0}(-1)}{1+x} .
$$

Thus,

$$
\tilde{S}_{0}^{1}(x)=\varphi_{0}(-1)-\frac{\varepsilon \varphi_{0}(-1)}{(1+x) \gamma_{, \varphi}}=\varphi_{0}(-1)-\frac{\varphi_{0}(-1)}{x^{\prime} \gamma_{, \varphi}} .
$$

It may be noted, too, that

$$
S_{1}^{0}\left(x^{\prime}\right)=-\frac{\varphi_{0}(-1)}{x^{\prime} \gamma_{, \varphi}}
$$

from (5.7), so that consistency with the asymptotic matching principle (3.5) is confirmed, with $n=1$ and $m=0$.

Finally, $\widetilde{S}_{1}^{1}(x)$ can be anticipated by applying (3.5) with $m=n=1$. The result, written in terms of $x^{\prime}$, is

$$
\begin{aligned}
\tilde{S}_{1}^{1}(x) \gamma_{, \varphi}= & \varepsilon x^{\prime}\left[\psi^{\prime}(-1)-\gamma_{, x}\left(-1, \varphi_{0}(-1)\right)\right]+\varepsilon \varphi_{0}^{\prime}(-1) \ln \left(\frac{2}{\varepsilon x^{\prime}}\right) \\
& +\varepsilon A+\varepsilon \varphi_{0}(-1)\left[\gamma_{, x \varphi}+\varphi_{0}^{\prime}(-1) \gamma_{, \varphi \varphi}\right] / \gamma_{, \varphi} .
\end{aligned}
$$

It is now possible to obtain an equation for $\tilde{\varphi}_{1}\left(x^{\prime}\right)$, by putting $x=-1+\varepsilon x^{\prime}$ in (2.1) and retaining terms up to order $\varepsilon$. Thus.

$$
\begin{aligned}
& -\varepsilon \int_{-1}^{1} \frac{d y}{(y-x)^{2}}\left[\varphi_{0}(y)+\varphi_{1}(y)+\tilde{\varphi}_{0}\left(y^{\prime}\right)+\tilde{\varphi}_{1}\left(y^{\prime}\right)-S_{0}^{1}\left(y^{\prime}\right)-S_{1}^{1}\left(y^{\prime}\right)\right] \\
& \quad+\gamma\left(-1+\varepsilon x^{\prime}, \tilde{\varphi}_{0}\left(x^{\prime}\right)+\tilde{\varphi}_{1}\left(x^{\prime}\right)\right) \sim \psi\left(-1+\varepsilon x^{\prime}\right) .
\end{aligned}
$$

Note that (5.12) shows that $\tilde{\varphi}_{1}\left(y^{\prime}\right)$ contains a term linear in $y^{\prime}$, as $y^{\prime} \rightarrow \infty$; therefore, terms in (5.13) are grouped, so that

$$
\begin{aligned}
& -\int_{0}^{\infty} \frac{d y^{\prime}}{\left(y^{\prime}-x^{\prime}\right)^{2}}\left[\tilde{\varphi}_{1}\left(y^{\prime}\right)-\varepsilon y^{\prime} \varphi_{0}^{\prime}(-1)\right]+\int_{2 / \varepsilon}^{\infty} \frac{d y^{\prime}}{\left(y^{\prime}-x^{\prime}\right)^{2}}\left[\tilde{\varphi}_{1}\left(y^{\prime}\right)-\varepsilon y^{\prime} \varphi_{0}^{\prime}(-1)\right] \\
& -\int_{0}^{\infty} \frac{d y^{\prime}}{\left(y^{\prime}-x^{\prime}\right)^{2}} \tilde{\varphi}_{0}\left(y^{\prime}\right)+\int_{2 / \varepsilon}^{\infty} \frac{d y^{\prime}}{\left(y^{\prime}-x^{\prime}\right)^{2}} \tilde{\varphi}_{0}\left(y^{\prime}\right) \\
& -\varepsilon \int_{-1}^{1} \frac{d y}{(y-x)^{2}}\left[\varphi_{0}(y)+\varphi_{1}(y)-\varphi_{0}(-1)-S_{1}^{1}\left(\frac{1+y}{\varepsilon}\right)\right] \\
& +\gamma\left(-1, \tilde{\varphi}_{0}\left(x^{\prime}\right)\right)+\tilde{\varphi}_{1}\left(x^{\prime}\right) \gamma_{. \varphi}\left(-1, \tilde{\varphi}_{0}\left(x^{\prime}\right)\right)+\varepsilon x^{\prime} \gamma_{. .}\left(-1, \tilde{\varphi}_{0}\left(x^{\prime}\right)\right) \\
& \sim \psi(-1)+\varepsilon x^{\prime} \psi^{\prime}(-1) .
\end{aligned}
$$


Equation (5.14) can be simplified as follows. First, Eq. (4.7) governing $\tilde{\varphi}_{0}$ eliminates three terms. Next, when $y^{\prime}>\frac{2}{\varepsilon}, \tilde{\varphi}_{0}\left(y^{\prime}\right) \sim \widetilde{S}_{0}^{1}(y)$, and $\tilde{\varphi}_{1}\left(y^{\prime}\right) \sim \widetilde{S}_{1}^{1}(y)$. With these substitutions, the integrals can be evaluated. The one involving $\tilde{\varphi}_{0}$ contributes $\frac{\varepsilon}{2} \varphi_{0}(-1)$ and the other contributes zero. Finally, consider the integral involving $\varphi_{0}$ and $\varphi_{1}$. The term $\varphi_{1}(y)-S_{1}^{1}\left(\frac{1+y}{\varepsilon}\right)=O(\varepsilon)$ uniformly and so contributes zero to (5.14), to order $\varepsilon$. The remaining integral can be evaluated, using (2.3), to give

$$
\varepsilon \int_{-1}^{1} \frac{d y}{(y-x)^{2}}\left[\varphi_{0}(y)-\varphi_{0}(-1)\right] \sim \varepsilon \varphi_{0}^{\prime}(-1) \ln \left(\frac{2}{\varepsilon x^{\prime}}\right)+\varepsilon A+\frac{\varepsilon}{2} \varphi_{0}(-1) .
$$

The resulting simplification of (5.14) is as follows:

$$
\begin{aligned}
-\int_{0}^{\infty} & \frac{d y}{\left(y^{\prime}-x^{\prime}\right)^{2}}\left[\tilde{\varphi}_{1}\left(y^{\prime}\right)-\varepsilon y^{\prime} \varphi_{0}^{\prime}(-1)\right]+\tilde{\varphi}_{1}\left(x^{\prime}\right) \gamma_{, \varphi}\left(-1, \tilde{\varphi}_{0}\left(x^{\prime}\right)\right) \\
& =\varepsilon x^{\prime}\left[\psi^{\prime}(-1)-\gamma_{, x}\left(-1, \tilde{\varphi}_{0}\left(x^{\prime}\right)\right)\right]+\varepsilon \varphi_{0}^{\prime}(-1) \ln \left(\frac{2}{\varepsilon x^{\prime}}\right)+\varepsilon A .
\end{aligned}
$$

This is a linear equation for $\tilde{\varphi}_{1}$.

It is desirable now to check the consistency of (5.16) with the asymptotic matching principle (3.5) which was assumed in its derivation. That is, it should follow from (5.16) that $\widetilde{S}_{1}^{1}(x)$ is indeed given by (5.12). To this end, let $\chi\left(x^{\prime}\right)$ be any smooth function with $\chi(0)=0$ and $\chi\left(x^{\prime}\right) \rightarrow 1$ as $x^{\prime} \rightarrow \infty$ and define

$$
\bar{\varphi}_{1}\left(x^{\prime}\right)=\tilde{\varphi}_{1}\left(x^{\prime}\right)-\varepsilon \chi\left(x^{\prime}\right) \varphi_{0}^{\prime}(-1) \ln \left(\frac{2}{\varepsilon x^{\prime}}\right) / \gamma_{, \varphi}\left(-1, \varphi_{0}(-1)\right)-\varepsilon x^{\prime} \varphi_{0}^{\prime}(-1) .
$$

Then (5.16) gives

$$
\begin{aligned}
-\int_{0}^{\infty} & \frac{d y^{\prime}}{\left(y^{\prime}-x^{\prime}\right)^{2}} \bar{\varphi}_{1}\left(y^{\prime}\right)+\bar{\varphi}_{1}\left(x^{\prime}\right) \gamma_{, \varphi}\left(-1, \tilde{\varphi}_{0}\left(x^{\prime}\right)\right) \\
= & \frac{\varepsilon \varphi_{0}^{\prime}(-1)}{\gamma_{, \varphi}\left(-1, \varphi_{0}(-1)\right)} \int_{0}^{\infty} \frac{d y^{\prime}}{\left(y^{\prime}-x^{\prime}\right)^{2}} \chi\left(y^{\prime}\right) \ln \left(\frac{2}{\varepsilon y^{\prime}}\right) \\
& \quad-\varepsilon \varphi_{0}^{\prime}(-1)\left[\chi\left(x^{\prime}\right) \frac{\gamma_{, \varphi}\left(-1, \tilde{\varphi}_{0}\left(x^{\prime}\right)\right)}{\gamma_{, \varphi}\left(-1, \varphi_{0}(-1)\right)}-1\right] \ln \left(\frac{2}{\varepsilon x^{\prime}}\right)+\varepsilon A \\
& \quad+\varepsilon x^{\prime}\left[\psi^{\prime}(-1)-\gamma_{, x}\left(-1, \tilde{\varphi}_{0}\left(x^{\prime}\right)\right)-\varphi_{0}^{\prime}(-1) \gamma_{, \varphi}\left(-1, \tilde{\varphi}_{0}\left(x^{\prime}\right)\right)\right] .
\end{aligned}
$$

When $x^{\prime} \rightarrow \infty$, the right side of (5.18) tends to the value

$$
\varepsilon A+\varepsilon \varphi_{0}(-1)\left[\gamma_{, x \varphi}+\varphi_{0}^{\prime}(-1) \gamma_{, \varphi}\right] / \gamma_{, \varphi},
$$

with $\gamma_{, x \varphi}$ etc. evaluated at $\left(-1, \varphi_{0}(-1)\right)$. It is therefore consistent that $\gamma_{, \varphi} \bar{\varphi}_{1}$ tends to the constant value (5.19), since then the integral on the left side of (5.18) tends to zero. Thus, it is consistent that

$$
\tilde{\varphi}_{1}\left(\frac{1+x}{\varepsilon}\right) \sim \tilde{S}_{1}^{1}(x)
$$

with $\widetilde{S}_{1}^{1}$ given by (5.12). 
6. Solution to second order: illustrative example. The solution as so far developed is formal in the sense that $\varphi_{0}$ and $\tilde{\varphi}_{0}$ have not been found explicitly. Also, to order $\varepsilon$, there is no interaction between the two end-points $x= \pm 1$. This appears first at order $\varepsilon^{2}$, as will now be illustrated. Very long expressions are avoided by restricting attention to the special case

$$
-\varepsilon \int_{-1}^{1} \frac{d y}{(y-x)^{2}} \varphi(y)+\varphi(x)=1, \quad-1<x<1 .
$$

This was considered to leading order by Hori and Nemat-Nasser [5] and Olmstead and Gautesen [6].

First, for Eq. (6.1), the solution to first order simplifies drastically, since $\gamma_{, \varphi}=1$ and all other derivatives of $\gamma$ are zero. The results of the preceding sections reduce to

$$
\begin{gathered}
\varphi_{0}(x)=1, \\
\varphi_{1}(x)=-\frac{2 \varepsilon}{1-x^{2}}
\end{gathered}
$$

and $\tilde{\varphi}_{0}, \tilde{\varphi}_{1}$ satisfy the equations

$$
\begin{gathered}
-\int_{0}^{\infty} \frac{d y^{\prime}}{\left(y^{\prime}-x^{\prime}\right)^{2}} \tilde{\varphi}_{0}\left(y^{\prime}\right)+\tilde{\varphi}_{0}\left(x^{\prime}\right)=1, \\
-\int_{0}^{\infty} \frac{d y^{\prime}}{\left(y^{\prime}-x^{\prime}\right)^{2}} \tilde{\varphi}_{1}\left(y^{\prime}\right)+\tilde{\varphi}_{1}\left(x^{\prime}\right)=-\varepsilon / 2 .
\end{gathered}
$$

Thus, for this particular problem

$$
\tilde{\varphi}_{1}\left(y^{\prime}\right)=-\frac{\varepsilon}{2} \tilde{\varphi}_{0}\left(y^{\prime}\right) .
$$

An explicit solution of (6.4) was first found by Koiter [11]. An alternative representation, better for present purposes, was developed by Olmstead and Gautesen [6]; it is summarized in the Appendix. The normalization used in [6] and retained in the Appendix is different from that employed in (6.4) but this can be adjusted by scaling the variables. The result is

$$
\tilde{\varphi}_{0}\left(x^{\prime}\right)=V_{1}\left(\frac{x^{\prime}}{\pi}\right),
$$

where

$$
V_{1}(\eta)=\int_{0}^{\eta} \nu(\sigma) d \sigma=1-\int_{\eta}^{\infty} \nu(\sigma) d \sigma
$$

with

$$
\nu(\eta)=\frac{1}{\pi} \int_{0}^{\infty} \frac{t d t}{\left(1+t^{2}\right)^{3 / 4}} \exp \left[-\eta t-\frac{1}{\pi} \int_{0}^{t} \frac{\ln r d r}{1+r^{2}}\right] .
$$

It follows from (6.9) that, as $\eta \rightarrow \infty$,

$$
\nu(\eta) \sim \frac{1}{\pi \eta^{2}}-\frac{\beta}{\pi^{2} \eta^{3}}+\frac{\ln (\eta)}{\pi^{2} \eta^{3}}
$$


where

$$
\beta=1+2 \int_{0}^{\infty} \ln (u) e^{-u} d u
$$

or, in terms of Euler's constant $\gamma=0.5772 \ldots$,

$$
\beta=1-2 \gamma
$$

Hence,

$$
\widetilde{S}_{0}^{2}(x)=1-\frac{\varepsilon}{1+x}-\frac{\varepsilon^{2} \gamma}{(1+x)^{2}}-\frac{\varepsilon^{2}}{(1+x)^{2}} \ln \left(\frac{1+x}{\pi \varepsilon}\right) .
$$

Note that $\widetilde{S}_{0}^{1}(x)$ is given by (6.12) with the term of order $\varepsilon^{2}$ removed. This is consistent with (5.10). The term of order $\varepsilon^{2}$ would be difficult to extract from the integral equation (6.4), in the absence of its explicit solution. It follows also from (6.6) and (6.12) that

$$
\widetilde{S}_{1}^{2}(x)=-\frac{\varepsilon}{2}+\frac{\varepsilon^{2}}{2(1+x)}
$$

From (6.2) and (6.3),

$$
\begin{gathered}
S_{0}^{2}\left(x^{\prime}\right)=1, \\
S_{1}^{2}\left(x^{\prime}\right)=-\frac{1}{x^{\prime}}-\frac{\varepsilon}{2}-\frac{\varepsilon^{2}}{4} x^{\prime} .
\end{gathered}
$$

The calculation of $\varphi_{2}$ and $\tilde{\varphi}_{2}$ now follows the pattern established in the preceding sections, although explicit allowance has to be made for the two end-points simultaneously. First, $\varphi_{2}$ is found by replacing the integrand in (6.1) by its uniform expansion, correct to order $\varepsilon$. Routine calculation then gives

$$
\begin{aligned}
\varphi_{2}(x)=\varepsilon^{2}\{ & -\frac{\gamma}{2}\left[\frac{1}{(1+x)^{2}}+\frac{1}{(1-x)^{2}}\right] \\
& -\frac{1}{(1-x)^{2}} \ln \left(\frac{1+x}{\pi \varepsilon}\right)-\frac{1}{(1-x)^{2}} \ln \left(\frac{1-x}{\pi \varepsilon}\right) \\
& \left.+\frac{2}{1-x^{2}}-\frac{1}{(1-x)^{2}} \ln \left(\frac{2}{1-x}\right)-\frac{1}{(1-x)^{2}} \ln \left(\frac{2}{1+x}\right)\right\}
\end{aligned}
$$

and hence, by expanding,

$$
\begin{aligned}
S_{2}^{2}\left(x^{\prime}\right)= & -\frac{1}{x^{\prime 2}} w\left\{\gamma+\ln \left(\frac{x^{\prime}}{\pi}\right)\right\}+\frac{\varepsilon}{2 x^{\prime}}-\frac{\varepsilon^{2}}{4} \ln \left(\frac{2}{\varepsilon x^{\prime}}\right) \\
& -\frac{\varepsilon^{2}}{4} \ln \left(\frac{2}{\pi \varepsilon}\right)+\left(\frac{3-2 \gamma}{8}\right) \varepsilon^{2} .
\end{aligned}
$$

Assuming the validity of the matching principle (3.5), it follows that

$$
\tilde{S}_{2}^{2}(x)=-\frac{\varepsilon}{4}(1+x)-\frac{\varepsilon^{2}}{4} \ln \left(\frac{2}{1+x}\right)-\frac{\varepsilon^{2}}{4} \ln \left(\frac{2}{\pi \varepsilon}\right)+\varepsilon^{2} \frac{(3-2 \gamma)}{8} .
$$


An equation for $\tilde{\varphi}_{2}\left(x^{\prime}\right)$ can now be formulated, by substituting into the integral in (6.1) a uniform approximation, correct to order $\varepsilon^{2}$. Its reduction is laborious but leads to the equation

$$
\begin{gathered}
-\int_{0}^{\infty} \frac{d y^{\prime}}{\left(y^{\prime}-x^{\prime}\right)^{2}}\left[\tilde{\varphi}_{2}\left(y^{\prime}\right)+\frac{\varepsilon^{2}}{4} y^{\prime}\right]+\tilde{\varphi}_{2}\left(x^{\prime}\right)+\frac{\varepsilon^{2}}{4} x^{\prime} \\
=\varepsilon^{2} \frac{(3-2 \gamma)}{8}-\frac{\varepsilon^{2}}{4} \ln \left(\frac{2}{\varepsilon \pi}\right)-\frac{\varepsilon^{2}}{4} \ln \left(\frac{2}{\varepsilon x^{\prime}}\right) .
\end{gathered}
$$

It is immediately apparent that the solution $\tilde{\varphi}_{2}$ has the asymptotic form (6.18), consistently with the asymptotic matching principle. In detail, it is expressible in terms of the two solutions developed in the Appendix. Thus,

$$
\tilde{\varphi}_{2}\left(x^{\prime}\right)+\frac{\varepsilon^{2}}{4} x^{\prime}=\frac{\varepsilon^{2}}{8}\left[3-2 \gamma-4 \ln \left(\frac{2}{\pi \varepsilon}\right)\right] \tilde{\varphi}_{0}\left(x^{\prime}\right)+\frac{\varepsilon^{2}}{4} V_{2}\left(\frac{x^{\prime}}{\pi}\right),
$$

where the Fourier transform of $V_{2}(\eta)$ is given by Eq. (A.18).

To second order, therefore,

$$
\tilde{\varphi}\left(x^{\prime}\right) \sim\left\{1-\frac{\varepsilon}{2}+\frac{\varepsilon^{2}}{8}\left[3-2 \gamma-4 \ln \left(\frac{2}{\pi \varepsilon}\right)\right]\right\} \tilde{\varphi}_{0}\left(x^{\prime}\right)+\frac{\varepsilon^{2}}{4}\left(V_{2}\left(\frac{x^{\prime}}{\pi}\right)-x^{\prime}\right) .
$$

The behaviour of (6.21) as $x^{\prime} \rightarrow 0$ follows from (A.12) and (A.24):

$$
\tilde{\varphi}\left(x^{\prime}\right) \sim \frac{2}{\pi}\left\{1-\frac{\varepsilon}{2}-\frac{\varepsilon^{2}}{8}\left[1+4 \gamma+4 \ln \left(\frac{2}{\pi \varepsilon}\right)\right]\right\}\left(x^{\prime}\right)^{1 / 2} .
$$

In terms of fracture mechanics, the coefficient of $\left(x^{\prime}\right)^{1 / 2}$ is proportional to the stress intensity factor at the tip of the crack. The term in brackets in (6.22) thus represents the ratio of the stress intensity factor for a bridged crack of finite length to the corresponding factor for a long crack $(\varepsilon \rightarrow 0)$.

Appendix: solution of a Wiener-Hopf equation. This appendix gives an outline of the solution of the integral equation

$$
-\frac{1}{\pi} \int_{0}^{\infty}(\sigma-\eta)^{-2} V(\sigma) d \sigma+V(\eta)=W(\eta), \quad \eta>0 .
$$

Eqs. (6.4) and (6.19) can be placed in this form by means of the variable changes $x^{\prime}=\pi \eta, y^{\prime}=\pi \sigma$. The special case $W(\eta)=1$ was discussed by Olmstead and Gautesen [6]. Following the method of [6], (A.1) is Fourier transformed to give

$$
(1+|\alpha|) \widehat{V}_{+}(\alpha)=\widehat{Q}_{-}(\alpha)+\widehat{W}_{+}(\alpha),
$$

where

$$
\widehat{V}_{+}(\alpha)=\int_{0}^{\infty} e^{i \ll \eta} V(\eta) d \eta
$$

$\widehat{W}_{+}(\alpha)$ is defined similarly and $\widehat{Q}_{-}(\alpha)$ is the Fourier transform of the (unknown) extension of (A.1) for $\eta<0$. Transforms with suffix " + " are analytic functions of $\alpha$ for $\operatorname{Im}(\alpha)>0$; when $\alpha$ is real, they are interpreted as boundary values as $\operatorname{Im}(\alpha) \downarrow 0$ of these analytic functions. Correspondingly, $\hat{Q}_{-}(\alpha)$ is analytic for $\operatorname{Im}(\alpha)<0$. 
Still following [6], the decomposition

$$
1+|\alpha|=K_{+}(\alpha) / K_{-}(\alpha)
$$

is employed (with $\alpha$ real), where

$$
K_{ \pm}(\alpha)=\exp \left\{ \pm \frac{1}{\pi} \int_{0}^{\infty} \frac{\ln (t \mp i \alpha)}{t^{2}+1} d t\right\} .
$$

Equation (A.2) can then be given in the form of a Hilbert problem:

$$
F_{+}(\alpha)-F_{-}(\alpha)=K_{-}(\alpha) \widehat{W}_{+}(\alpha),
$$

where

$$
\begin{aligned}
F(\zeta) & =K_{+}(\zeta) \widehat{V}_{+}(\zeta), & \operatorname{Im}(\zeta)>0 \\
& =K_{-}(\zeta) \widehat{Q}_{-}(\zeta), & \operatorname{Im}(\zeta)<0 .
\end{aligned}
$$

A solution of (A.6) is

$$
F(\zeta)=\frac{1}{2 \pi i} \int_{-\infty}^{\infty} \frac{K_{-}(\alpha) \widehat{W}_{+}(\alpha) d \alpha}{\alpha-\zeta}
$$

This differs from the general solution by an entire function which will, in fact, have to be zero in the examples considered.

(a) $W(\eta)=1$. When $W(\eta)=1, \widehat{W}_{+}(\alpha)=i \alpha^{-1}$ when $\operatorname{Im}(\alpha)>0$, so that

$$
\widehat{W}_{+}(\alpha)=i(\alpha+0 i)^{-1}
$$

when $\alpha$ is real. Then, from (A.8), when $\operatorname{Im}(\zeta)>0$, the integral can be evaluated by closing the contour in the lower half-plane and using Cauchy's theorem: the only contribution is from the pole at the origin. Thus

$$
K_{+}(\zeta) \widehat{V}_{+}(\zeta)=i K_{-}(0) / \zeta
$$

or

$$
\widehat{V}_{+}(\zeta)=\frac{i}{\zeta K_{+}(\zeta)}
$$

since $K_{-}(0)=1$.

Note that $K_{+}(\zeta) \sim(-i \zeta)^{1 / 2}$ as $\zeta \rightarrow \infty$, so $\widehat{V}_{+}(\zeta) \sim(i)^{3 / 2} \zeta^{-3 / 2}$. Correspondingly,

$$
V(\eta) \sim 2 \pi^{-1 / 2} \eta^{1 / 2} \text { as } \eta \rightarrow 0 .
$$

Olmstead and Gautesen [6] developed the solution $V_{1}(\eta)$ given by $(6.8),(6.9)$ from the Fourier transform (A.11). They also deduced (A.12), directly from $V_{1}(\zeta)$.

(b) $W(\eta)=\ln (\eta)$. In this case, when $\operatorname{Im}(\alpha)>0$,

$$
\widehat{W}_{+}(\alpha)=\frac{i}{\alpha}\left[\frac{i \pi}{2}-\gamma-\ln \alpha\right],
$$

Euler's constant $\gamma$ arising from the integral

$$
\int_{0}^{\infty} \ln (x) e^{-x} d x=-\gamma
$$


It follows from (A.8) that, when $\operatorname{Im}(\zeta)>0$,

$$
K_{+}(\zeta) \widehat{V}_{+}(\zeta)=\frac{1}{2 \pi} \int_{-\infty}^{\infty} \frac{K_{-}(\alpha)[i \pi / 2-\gamma-\ln (\alpha+0 i)]}{(\alpha+0 i)(\alpha-\zeta)} d \alpha
$$

so

$$
\begin{aligned}
K_{+}(\zeta) \widehat{V}_{+}(\zeta)= & \frac{1}{2 \pi} \int_{-\infty}^{\infty} \frac{\left(K_{-}(\alpha)-1\right)[i \pi / 2-\gamma-\ln (\alpha+0 i)]}{\alpha(\alpha-\zeta)} d \alpha \\
& +\frac{i}{\zeta}\left[\frac{i \pi}{2}-\gamma-\ln \zeta\right] .
\end{aligned}
$$

The extra terms in (A.16), in comparison with (A.15), add up to zero: this can be verified by use of Cauchy's theorem. The integral in (A.16) can be simplified by deforming the contour so that it wraps around the branch cut of the logarithm. Thus,

$$
K_{+}(\zeta) \widehat{V}_{+}(\zeta)=-i \int_{-\infty}^{0} \frac{\left(K_{-}(\alpha)-1\right) d \alpha}{\alpha(\alpha-\zeta)}+\frac{i}{\zeta}\left[\frac{i \pi}{2}-\gamma-\ln \zeta\right]
$$

or, equivalently,

$$
\begin{aligned}
K_{+}(\zeta) \widehat{V}_{+}(\zeta)= & -i \int_{-\infty}^{-1} \frac{K_{-}(\alpha) d \alpha}{\alpha(\alpha-\zeta)}-i \int_{-1}^{0} \frac{\left(K_{-}(\alpha)-1\right)}{\alpha(\alpha-\zeta)} \\
& +\frac{i}{\zeta}\left[\frac{i \pi}{2}-\gamma-\ln \left(\frac{\zeta}{1+\zeta}\right)\right] .
\end{aligned}
$$

The function $V(\eta)$ whose Fourier transform is defined by (A.18) is referred to as $V_{2}(\eta)$ in the text.

The asymptotic form of $\widehat{V}_{+}(\zeta)$ as $\zeta \rightarrow \infty$ is readily deduced from (A.18):

$$
\widehat{V}_{+}(\zeta) \sim(i)^{3 / 2} k j \zeta^{-3 / 2}
$$

where

$$
k=\frac{i \pi}{2}-\gamma+\int_{-\infty}^{-1} \frac{K_{-}(\alpha) d \alpha}{\alpha}+\int_{-1}^{0} \frac{\left(K_{-}(\alpha)-1\right) d \alpha}{\alpha} .
$$

Hence,

$$
V_{2}(\eta) \sim 2 \pi^{-1 / 2} k \eta^{1 / 2} \quad \text { as } \eta \rightarrow 0 .
$$

That the constant $k$ is real can be verified by calculating its imaginary part, using

$$
\overline{K_{-}(\alpha)}=K_{-}(\alpha) \text {. }
$$

Computation of the integrals in (A.20) confirms this to high accuracy and, furthermore, suggests that

$$
k=-(1+\gamma),
$$

exactly. This has not been verified by analysis but the result (which at least is a highly accurate approximation), that

$$
V_{2}(\eta) \sim-2 \pi^{-1 / 2}(1+\gamma) \eta^{1 / 2} \quad \text { as } \eta \rightarrow 0
$$

is used in the text. 
Acknowledgments. Thanks are due to Dr. L. Ni (UCSD) for checking the algebra, and to R. J. Bedding (Bath) for computing the integrals in (A.20). Most of the work was performed during a visit by JRW to UCSD. The support of the U. S. Army Research Office under Contract Number DAAL-03-86-K-0169 is gratefully acknowledged.

\section{REFERENCES}

[1] J. S. Angell and W. E. Olmstead, Singularly perturbed Volterra integral equations, SIAM J. Appl. Math. 47, 1-14 (1987)

[2] J. S. Angell and W. E. Olmstead, Singularly perturbed Volterra integral equations II, SIAM J. Appl. Math. 47, 1150-1162 (1987)

[3] C. G. Lange and D. R. Smith, Singular perturbation analysis of integral equations, Stud. Appl. Math. 79, 1-63 (1988)

[4] C. Atkinson and F. G. Leppington, The asymptotic solution of some integral equations, IMA J. Appl. Math. 31, 169-182 (1983)

[5] M. Hori and S. Nemat-Nasser, Asymptotic solution of a class of strongly singular integral equations, SIAM J. Appl. Math. (to appear)

[6] W. E. Olmstead and A. K. Gautesen, Asymptotic solution of some singularly perturbed Fredholm equations, Z. Angew. Math. Phys. 40, 230-244 (1989)

[7] M. D. Van Dyke, Perturbation Methods in Fluid Mechanics, Academic Press, New York, 1964 (also: Perturbation Methods in Fluid Mechanics (annotated edition), The Parabolic Press, Palo Alto (1975))

[8] J. Hadamard, Lectures on Cauchy's Problem in Linear Partial Differential Equations, Dover, New York, 1952

[9] I. M. Gel' fand and G. E. Shilov, Generalized Functions, Volume 1. Properties and Operations, Academic Press, New York, 1964

[10] S. Nemat-Nasser and M. Hori, Toughening by partial or full bridging of cracks in ceramics and fiber reinforced composites, Mech. Mat. 6, 245-269 (1987)

[11] W. T. Koiter, On the diffusion of load from a stiffener into a sheet, Quart. J. Mech. Appl. Math. 8, 164-178 (1955) 